\title{
NÍVEL DE CONHECIMENTO E DESIDRATAÇÃO DE JOGADORES JUNIORES DE FUTEBOL
}

LEVEL OF KNOWLEDGE AND DEHYDRATION OF JUNIOR SOCCER PLAYERS

NIVEL DE CONOCIMIENTO Y DESHIDRATACIÓN EN JUGADORES JUVENILES DE FÚTBOL

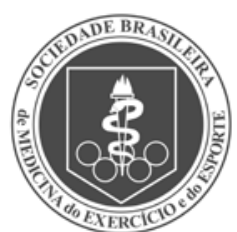

Artigo Original

Original ARticle Artículo Original
Vanessa Machado Lustosa' (Nutricionista)

Fátima Karina Costa de Araújo ${ }^{2}$ (Nutricionista)

Henrilla Mairla Santos de Morais² (Nutricionista)

Fabiane Araújo Sampaio²

(Nutricionista, Professora Assistente)

1. Faculdade Santo Agostinho,

Programa de Pós Graduação em Nutrição Clínica de Funcional,

Teresina, PI, Brasil.

2. Faculdade de Ciências e

Tecnologia do Maranhão, Caxias, MA, Brasil.

\section{Correspondência:}

Fabiane Araújo Sampaio

Rua Dr. Nathan portela Nunes, 4127, apto 7, Ininga, Teresina, PI, Brasil. 64048-495.

fabianesampaio21@gmail.com

\section{RESUMO}

Introdução: A desidratação é uma condição fisiológica que decorre de perda hídrica prolongada, com consequente elevação da temperatura corporal e redução do desempenho dos atletas. Objetivo: Avaliar o nível de conhecimento e o grau de hidratação de jogadores juniores de futebol. Métodos: Trata-se de um estudo transversal, envolvendo 14 jogadores de futebol, que foram avaliados quanto ao percentual de perda de peso após exercício (desidratação relativa), pH e densidade da urina e nível de conhecimento sobre hidratação. A análise estatística foi realizada por meio do software SPSS versão 18.0, utilizando um intervalo de confiança de 95\%, com nível de significância com $p<0,05$. Resultados: Pôde-se verificar que 92,85\% dos jogadores apresentaram eutrofia e os valores médios de desidratação relativa, densidade urinária e pH foram de -0,89\%, 1.034,43, e 5,64, respectivamente, com o que se revelou desidratação para as posições com maior desgaste físico e 48,9\% dos jogadores apresentam conhecimento regular sobre hidratação. Conclusão: A desidratação encontrada em jogadores juniores pode ser influenciada por diferentes posições em campo e esquema tático do jogo.

Descritores: esportes; hidratação; desidratação.

\section{ABSTRACT}

Introduction: Dehydration is a physiological condition that results from prolonged water loss, with consequent elevation of body temperature and reduced athletes' performance. Objective: To evaluate the level of knowledge and the degree of hydration ofjunior soccer players. Methods: This is a cross-sectional study involving 14 soccer players who were evaluated for weight loss after exercise (relative dehydration), pH and density of urine and the level of knowledge about hydration. Statistical analysis was performed using SPSS software version 18.0, using a 95\% confidence interval, with significance level of $p<0.05$. Results: It was possible to verify that $92.85 \%$ of the players were eutrophic and the mean values of relative dehydration, urinary density and $\mathrm{pH}$ were of $-0.89 \%, 1,034.43$, and 5.64 , respectively, revealing dehydration for positions with greater physical wear and $48.9 \%$ of the players had a regular knowledge about hydration. Conclusion: The dehydration found in junior players can be influenced by different positions in the field and tactical scheme of the game.

Keywords: sports; fluid therapy; dehydration.

\section{RESUMEN}

Introducción: La deshidratación es una condición fisiológica que resulta de la pérdida prolongada de agua, con la consiguiente elevación de la temperatura corporal y la reducción de rendimiento de los atletas. Objetivo: Evaluar el nivel de conocimiento y el grado de hidratación de jugadores de fútbol juvenil. Métodos: Se trata de un estudio transversal que incluyó a 14 jugadores de fútbol, que fueron evaluados para el porcentaje de pérdida de peso después del ejercicio (deshidratación relativa), el pH y la densidad de la orina y el nivel de conocimiento sobre la hidratación. El análisis estadístico se realizó mediante el software SPSS versión 18.0 usando un intervalo de confianza de 95\%, con un nivel de significación de $p<0,05$. Resultados: Se verificó que 92,85\% de los jugadores eran eutróficos y los valores promedio de deshidratación relativa, densidad y $\mathrm{pH}$ urinario fueron -0,89\%, 1.034,43, y5,64, respectivamente, que reveló deshidratación en las posiciones con mayor desgaste físico y que el $48,9 \%$ de los jugadores tiene un conocimiento normal sobre la hidratación. Conclusión: La deshidratación encontrada en jugadores juveniles puede ser influenciada por diferentes posiciones en el campo y el juego táctico.

Descriptores: deportes; fluidoterapia; deshidratación.

\section{INTRODUÇÃO}

O futebol é um dos esportes que vem crescendo a cada ano, sendo um evento importante com repercussão no mundo esportivo. Essa modalidade é caracterizada por envolver duas equipes de 11 jogadores, sendo 10 atletas de linha e um goleiro, divididos em posições de defesa, meio de campo e de ataque $\mathrm{e}^{1,2}$.
Estudos têm demonstrado alguns impactos negativos, como a desidratação e hipertermia e ainda o aparecimento da diminuição do volume plasmático. Durante uma partida e treinamento contínuo a reidratação não é suficiente para compensar a água perdida devido ao calor gerado durante a atividade física. Além disso, condições ambientais, a inexistência de pausas regulares nos jogos para a ingestão de líquidos pode induzir a um quadro de desidratação 2,3. 
A desidratação é uma condição fisiológica que decorre de uma prolongada perda hídrica, o que afeta as funções fisiológicas e a temperatura corporal, levando a complicações e prejuízos no desempenho durante o exercício de atletas, onde as consequências podem ser diminuídas ou até evitadas por meio de uma adequada reposição hídrica.

Para isso, é necessária a ingestão de líquidos contendo eletrólitos e a suplementação de carboidratos antes e durante o exercício. Esta prática é extremamente benéfica para o jogador e deve ser considerada a individualidade e posição de cada jogador, a intensidade do exercício e situações climáticas para que seja minimizado os efeitos da desidratação ${ }^{5-7}$.

Sabendo que o Brasil é um país tropical de clima quente e baixa umidade, da relevância do tema e ainda da necessidade de educar técnicos e atletas a respeito da hidratação adequada justificaram a realização desse estudo, que teve como objetivo avaliar o grau de desidratação, e verificar o nível de conhecimento sobre hidratação desses atletas.

\section{MÉTODOS}

Estudo transversal, realizado em um Clube de Futebol na cidade de Caxias-Maranhão, com 14 jogadores entre 16 e 19 anos de idade.

Primeiramente foram avaliadas as condições climáticas por meio da temperatura e umidade relativa do ar, posteriormente foi coletado peso e altura dos participantes para o cálculo de índice de massa corpórea (IMC). Para a verificação do grau de hidrataçãofoi coletadaa massa corporal antes (MCpré) e após a sessão de treinamento (MCpós), e realizado o cálculo da Desidratação relativa: Desidratação Relativa = MCpré - Mcpós, onde o \% de variação de peso corporal +1 a-1 Kg, o atleta era considerado hipohidratado, -1 a-3 Kg, Desidratação mínima, -3 a-5 Kg Desidratação significativa, > -5 Kg, Desidratação grave ${ }^{8}$.

A urina foi coletada antes e após o treino, para a determinação da densidade por meio de um refratômetro óptico, e a determinação do pH da urina por meio de fitas de determinação de pH (Labtest Diagnostica S.A. ${ }^{\circledR}$ ). Os valores para determinar o estado de hidratação segundo a densidade da urina são: Hipohidratação = 1030 sg; Euhidratados = 1013 a 1029 sg; Hiperhidratação $=1001$ a 1012 sg .

Para a avaliação do conhecimento dos atletas sobre hidratação foi realizado um questionário (auto administrado) adaptado anteriormente empregado com atletas de outras modalidades ${ }^{9,10}$, o qual continha oito perguntas objetivas relacionadas aos hábitos e nível de conhecimento sobre a temática hidratação. Classificados com nível de conhecimento: ruim $=0$ a 30\%; regular $=>30$ a 50\%; bom $=>50$ a $70 \%$ e ótimo $=>70 \%$.

Os dados foram organizados em planilhas do Exce ${ }^{\circledR}$, posteriormente exportados para o programa SPSS (for Windows ${ }^{\circledR}$ versão 18.0) para análise descritiva e o teste de Mann- Whitney. Nas respectivas análises estatísticas, foi utilizado nível de significância de $p \leq 0,05$ e nível de confiança de $95 \%$. O projeto foi aprovado sob o número do CAAE 42339015.1.0000.5554 e todos os participantes assinaram um termo de consentimento livre esclarecido.

\section{RESULTADOS}

Participaram do estudo 14 jogadores de futebol juniores com média de idade de 17,07 \pm 1,07 anos, o que os classificam como adolescente, destes 92,85\% apresentaram eutrofia, segundo indicador IMC/Idade. Os valores de peso antes e após o treino para a determinação da desidratação relativa demonstraram que apenas os jogadores do lateral direito e esquerdo, volante e o zagueiro 3 estão desidratados, os demais jogadores apresentaram euhidratação

Quanto à densidade urinária antes e após o treino dos jogadores, verificou-se que apenas quatro jogadores apresentaram quadro de hidratação adequada antes de começar a partida, sendo eles o goleiro, o zagueiro 2, o atacante e o volante, os demais jogadores antes de iniciar a partida já revelava desidratação. Vale destacar que o volante hidratado antes da partida, se desidratou ao final da mesma, segundo o método de densidade urinária.

Os valores médios e desvios padrão dos parâmetros densidade urinária pós-treino, desidratação relativa e o ph pré e pós-treinos estão descritos na Tabela 1, pôde-se observar que a desidratação relativa classifica os jogadores como Euhidratado, enquanto a densidade urinária pós-treino classifica-os como Hipohidratados, houve diferença estatística entre o pH antes e após a partida $(p<0,05)$, com redução do pH urinário. Enquanto que a temperatura, sensação térmica e umidade relativa do ar referente ao dia do treino da coleta de dados estão descritos na Tabela 2.

Na Tabela 3, é apresentado o percentual do nível de conhecimento dos jogadores avaliados, descritos de acordo com a ordem das perguntas. Quanto à preocupação dos jogadores em se hidratarem durante os treinos e competições foi observado que $64,28 \%$ e $71,42 \%$ dos atletas sempre realizam hidratação no momento do treino e durante as competições, respectivamente, porém $28,7 \%$ e $28,58 \%$ às vezes realizam hidratação no momento do treino e durante as competições e apenas 7,15\% quase nunca se hidratam durante o treino.

Quanto à preocupação dos jogadores com o tipo de hidratação (água ou isotônico), nos momentos que antecedem, durante ou depois de um treinamento ou competição foi demonstrado que 57,2\% dos jogadores se preocupam. Foi possível identificar todos os jogadores utilizam apenas como fonte de hidratação, sem a ingestão de outros tipos de bebidas.

Em relação ao momento ideal do consumo de líquido, 64,28\% dos jogadores declararam que a ingestão deve ser feita antes da sensação de sede, enquanto que $28,57 \%$ e 7,15\% acreditam que deve ser realizada somente após a sensação de sede ou muita sede, respectivamente.

Quando questionados sobre a importância da hidratação em diferentes estações do ano, o resultado revelou que 57,2\% se preocupavam com a hidratação independente da estação do ano e 42,8\% se preocupavam apenas no verão.

Na Figura 1 são descritos os principais sintomas apresentados pelos jogadores no momento do treino ou competições devido à desidratação e na Figura 2 é apresentado a relação entre volume de líquido adequado e a frequência que eles devem consumir. Quanto ao conhecimento dos jogadores sobre a função dos isotônicos apresentado na Figura 3, foi observado que $50 \%$ deles acertaram qual a principal função do isotônico.

Tabela 1. Valores médios e desvio padrão da densidade urinária pós-treino, desidratação relativa e o ph pré e pós-treino dos jogadores de futebol.

\begin{tabular}{c|c|c|c|c}
\hline Parâmetros & $\begin{array}{c}\text { Desidratação } \\
\text { relativa }\end{array}$ & $\begin{array}{c}\text { Densidade } \\
\text { urinária } \\
\text { pós-treino }\end{array}$ & pH pré-treino & pH pós-treino \\
\hline Média \pm DP & $-0,89 \pm 0,47$ & $1034,43 \pm 5,42$ & $5,93 \pm 0,82^{*}$ & $5,64 \pm 0,50^{*}$ \\
\hline Mínimo & -2 & 1024 & 5 & 5 \\
\hline Máximo & $-0,3$ & 1041 & 7 & 6 \\
\hline
\end{tabular}

Tabela 2. Distribuição dos valores de médios das variáveis referente as condições climáticas no dia do treinamento.

\begin{tabular}{c|c|c|c}
\hline Parâmetros & Temperatura & Sensação térmica & Umidade \\
\hline Média & $29^{\circ} \mathrm{C}$ & $36^{\circ} \mathrm{C}$ & $72,5 \%$ \\
\hline Mínimo & $23^{\circ} \mathrm{C}$ & $30^{\circ} \mathrm{C}$ & $50 \%$ \\
\hline Máximo & $35^{\circ} \mathrm{C}$ & $42^{\circ} \mathrm{C}$ & $95 \%$ \\
\hline Fonte: Dados da pesquisa.
\end{tabular}

Tabela 3. Percentual do Nível de conhecimento sobre hidratação.

\begin{tabular}{c|c|c}
\hline \multirow{2}{*}{ Nível de Conhecimento } & \multicolumn{2}{|c}{ Jogadores de Futebol } \\
\cline { 2 - 3 } & (n) & (\%) \\
\hline (\%) média de acerto das questões & 14 & $48,9 \%$ \\
\hline
\end{tabular}

Fonte: Dados da pesquisa. 


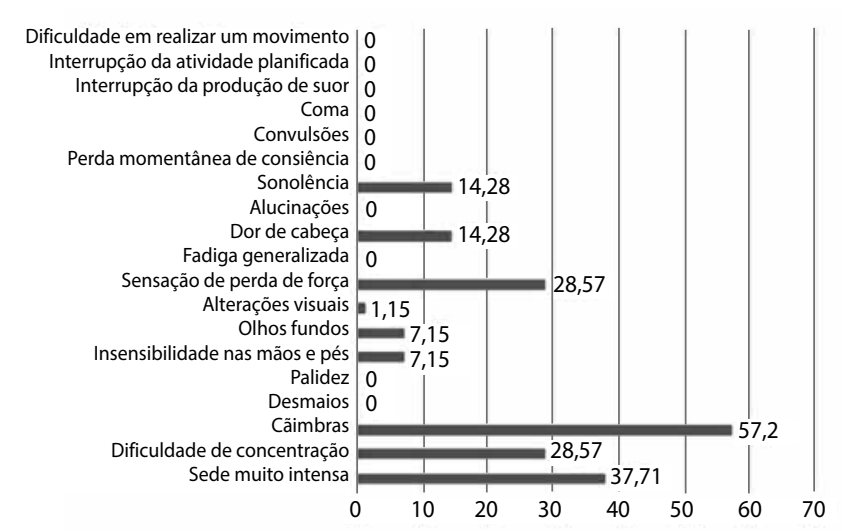

Figura 1. Sintomas relatados pelos jogadores decorrentes da desidratação.

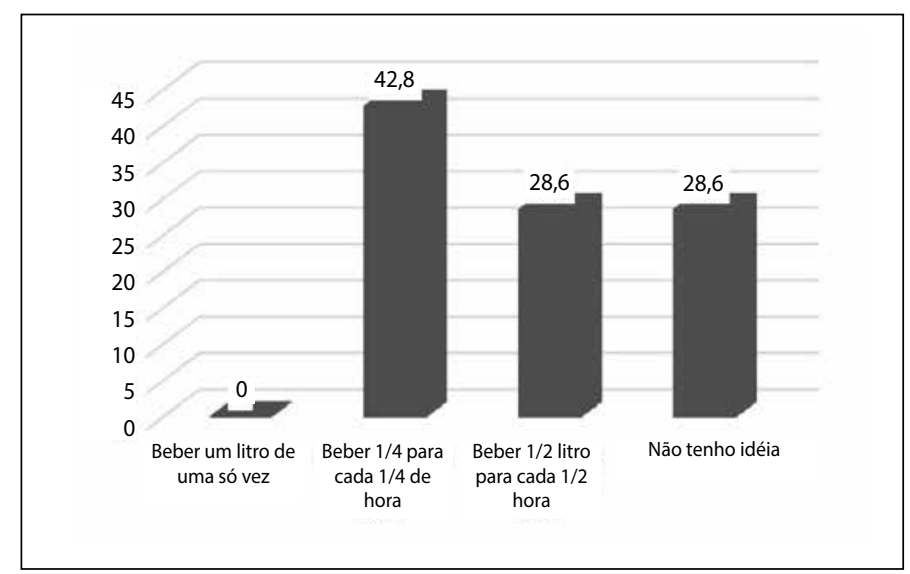

Figura 2. Relação entre tempo e quantidade de líquidos consumidos pelos jogadores.

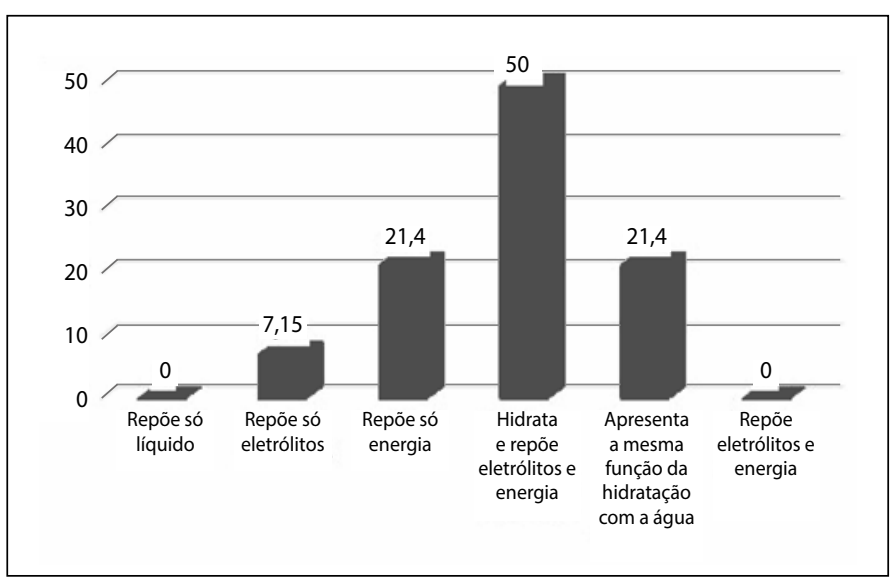

Figura 3. Conhecimento dos jogadores sobre a função dos isotônicos.

\section{DISCUSSÃO}

Neste estudo foi avaliado o grau de desidratação de jogadores de futebol juniores e o conhecimento destes sobre a hidratação. Pôde-se verificar que a maioria apresentou eutrofia segundo o indicador IMC por idade, condição favorecida pelo treino contínuo, resultados semelhantes foram encontrados em outros estudo ${ }^{11,12}$

Quanto avaliação do grau de desidratação, um dos métodos utilizados para a verificação foi aferição do peso pré e pós-treino, com determinação do percentual de Desidratação Relativa (DR\%) dos jogadores avaliados. Estes em sua maioria apresentaram a classificação Euhidratados, corroborando com os resultados de Ersoy et al. ${ }^{13}$, Motta e Quintão ${ }^{14}$, segundo os pesquisadores a temperatura e a umidade apresentavam condições favoráveis para a manutenção da hidratação em níveis aceitáveis. Por outro lado, o estudo de Mota et al. ${ }^{15}$ e Borusch et al. ${ }^{16}$ verificaram que a maioria dos atletas desidratou durante o treino, em virtude da elevada temperatura e da intensidade do treino naquele dia.

No presente estudo apenas o volante e os laterais direito e esquerdo demonstraram desidratação mínima, em virtude de serem posições mais suscetíveis ao desgaste físico e aumento de temperatura durante a partida. Segundo Ravagnani et al. ${ }^{17}$, os laterais precisam correr do campo de defesa até o campo de ataque e ainda realizam cruzamentos, o que favorece o aumento da produção de suor, com consequente desidratação.

Com relação à densidade urinária foram encontradas divergências com os dados da DR\%, a primeira diagnosticou os jogadores em estado de Hipohidratação antes de entrar em campo e apenas o goleiro, zagueiro 2 e o atacante permaneceram Euhidratados após o treino, jogadores de posições que realizam exclusivamente movimentos de curta duração e alta intensidade, recuperação passiva e baixo volume de deslocamento quando comparados aos outros atletas, conforme o esquema tático do clube avaliado, cujo sistema relatado foi o 4-3-3, caracterizado por conter quatro jogadores na defesa, três jogadores no meio-campo (com um ou dois volantes) e três jogadores no ataque ${ }^{18}$.

É importante ressaltar que ambos os métodos são válidos para verificar o estado de hidratação, entretanto, o peso antes e depois do treino avalia apenas mudanças rápidas na hidratação do atleta, fato este que pode ter influenciado os resultados encontrados. Nóbrega et al. ${ }^{4}$ também revelou que o estado Euhidratado para jogadores amadores de futsal por meio do método de DR\% e Hipohidratação, segundo a densidade urinária. Enquanto que os estudos de Nery et al. ${ }^{19}$, Pinto et al. ${ }^{20}$ e Ersoy et al. ${ }^{13}$, a DU não apresentou diferença significativa entre o pré e pós-treinamento.

Além disso, pôde-se verificar redução do pH urinário após o treino, decorrente principalmente do aumento na concentração da urina dos atleta investigados, logo também um indicador de desidratação. A urina naturalmente é ácida, com o pH entre 4,5 e 8, considerada adequada ligeiramente ácido com valores entre 5.5 a 6.5, superiores ao encontrado nesse estudo ${ }^{21}$.

As condições térmicas ambientais também podem ter influenciado a desidratação encontrada em grande parte dos jogadores, uma vez que o ambiente desfavorável ocasiona situações de estresse térmico e prejudica o desempenho das atividades humanas ${ }^{22}$. A temperatura elevada e a umidade relativa do ar diminuída no momento da análise pode ter prejudicado o desempenho dos jogadores.

Quanto ao nível de conhecimento sobre hidratação foi possível observar que os jogadores apresentaram um conhecimento intermediário e no que diz respeito ao hábito de se hidratar durante os treinamentos e competição, foi observado que grande parte dos avaliados sempre realiza hidratação. O resultado encontrado por Drumond et al..$^{23}$ demonstrou que apenas $50 \%$ dos participantes dos estudos consumiram líquidos sempre durante os treinos e competições.

Em relação à preocupação com tipo de hidratação (água ou isotônico) nos momentos que antecedem, durante e depois de um treinamento e competição, mais da metade dos jogadores reconhecem a importância, entretanto, 100\% dos atletas avaliados utilizavam apenas água para realizar sua hidratação, corroborando com estudos anteriores realizados em adultos e adolescentes futebolistas 24,25 .

Atividades com tempo inferior a 60 minutos de duração, a água é a bebida mais indicada, porém, atividades que ultrapassam esse período como uma partida de futebol que possui duração média de 90 minutos, a ingestão de apenas água é insuficiente para a hidratação dos jogadores. ASBME ${ }^{26}$ recomenda para atividades de duração superior a uma hora ou intensas do tipo intermitente mesmo com menos de uma hora a reposição 
de carboidrato e sódio, ou seja, a utilização de bebidas com 6\% de carboidrato passam a ser mais importante na recuperação do atleta, além de evitar quadros de hipoglicemia e acelerar a reposição do glicogênio muscular 7,27 .

No presente estudo a maioria dos jogadores se hidratam antes da sensação de sede, resultado semelhante foi encontrado por Prado et al. ${ }^{28}$, enquanto Ferreira et al. ${ }^{29}$ encontraram dados preocupantes em atletas de futebol de base, pois a maioria somente se hidratava após a sensação de sede. Assim, os resultados encontrados revelam a necessidade de sensibilizar os esportistas, uma vez que iniciar a ingestão de líquidos apenas após a sensação de sede pode induzir a sérios dados a saúde, pois a literatura afirma que quando o indivíduo sente sede, já apresenta 2\% de desidratação.

Ao se avaliar o hábito de hidratação dos jogadores em diferentes estações do ano, mais da metade realizam hidratação independente da estação. A desidratação é mais acentuada em dias quentes e úmidos, porém mesmo em situação de inverno, quando as médias térmicas estão mais baixas, existe possibilidade de desidratação, decorrente da baixa ingestão de líquidos associada a perda através de mecanismos de convecção e condução ${ }^{26}$.

Dentre os sintomas relatados pelos jogadores durante ou após o treino, grande parte relataram cãibras, seguido por sede muito intensa, dificuldade de concentração e sensação da perda de força. No estudo de Drumond et al. ${ }^{23}$ os sintomas mais frequentes foram sede muito intensa e cãibras, o primeiro indica que o jogador necessita repor seus estoques hídricos, uma vez que apresenta $2 \%$ de desidratação, enquanto que as cãibras também indicam desidratação e ainda concentrações anormais de eletrólitos séricos e estresse ambiental. Outros sinais como dificuldade na concentração e perda de força podem estar relacionados à presença de hipoglicemia ${ }^{30}$. Portanto, os resultados encontrados nesse estudo indicam que somente a água não foi suficiente para a hidratação dos jogadores avaliados e reforça a importância da reposição adequada hidroeletrolítica.

Além disso, deve ocorrer equilíbrio entre o volume e a frequência na ingestão de líquidos. Nossos jogadores apresentaram bom conhecimento sobre este tema, uma vez que quase metade dos atletas respondeu que o consumo de líquidos deve ser de $250 \mathrm{~mL}$ a cada 15 minutos, no entanto, o número de jogadores que não tinham ideia de como se hidratar ou não sabiam a informação adequada ainda é alarmante. A SBME ${ }^{26}$ recomenda que a ingestão de água deva ser duas horas antes do jogo em torno de 250 a $500 \mathrm{~mL}$ para garantir que o indivíduo inicie o exercício bem hidratado, enquanto que durante o exercício recomenda-se iniciar a ingestão nos primeiros 15 minutos e continuar bebendo a cada 15 a 20 minutos.

Em relação à função dos isotônicos, metade dos jogadores compreende o valor de seu uso, sabendo sua verdadeira função, apesar de nenhum deles realizarem a ingestão de bebida hidroeletrolítica. 0 modo mais conveniente e eficiente de reposição ocorre por meio de isotônicos, onde além de promoverem hidratação, apresentam ainda quantidades suficientes de eletrólitos e carboidratos ${ }^{30}$.

Nesse sentido, a desidratação presente em alguns jogadores desse estudo pode estar relacionada ao nível de conhecimento regular desses atletas ou mesmo decorrente da solicitação metabólica individual, o que reforça a necessidade de orientações sobre a temática para o grupo, visando corrigir os hábitos inadequados sobre hidratação.

\section{CONCLUSÃO}

O presente estudo permite concluir que a desidratação encontrada em jogadores juniores pode ser influenciada por diferentes posições em campo e esquema tático do jogo, uma vez que apenas atletas com funções de maior solicitação metabólica demonstra hidratação insuficiente. Além disso, os jogadores apresentam nível de conhecimento regular sobre hidratação, o que contribui para que alguns atletas iniciem partidas desidratados e apresentem sintomas característicos dessa condição, sugerindo a necessidade da utilização de isotônicos e não apenas água durante as atividades intensas e de duração superior a uma hora.

Todos os autores declararam não haver qualquer potencial conflito de interesses referente a este artigo.

CONTRIBUIÇÕES DOS AUTORES: Cada autor contribuiu individual e significativamente para o desenvolvimento do manuscrito. VML (0000-0003-0324-9517)* e FAS (0000-0002-9449-5045)*, foram as principais contribuintes na redação do manuscrito. VML, FKCA (0000-0002-4515-1684)* e HMSM (0000-0002-5360-4487)* realizaram e avaliaram a coleta de dados. FAS realizou a análise estatística. VML e FAS realizaram a pesquisa bibliográfica, a revisão do manuscrito e contribuíram com o conceito intelectual do estudo. ${ }^{*}$ ORCID (Open Researcher and Contributor ID).

\section{REFERÊNCIAS}

1. Reis $V A B$, Azevedo COE, ROSSI L. Perfil antropométrico e taxa de sudorese no futebol juvenil. Rev Bras Cineantropom Desempenho Hum. 2009;11(2):134-41

2. Rico-Sanz J. Body composition and nutritional assessments in soccer. Int J Sport Nutr. 1998;8(2):113-23.

3. Balikian P, Lourenção A, Ribeiro LFP, FestucciaWTL, Neiva CM. Consumo máximo de oxigênio e limiar anaeróbio de jogadores de futebol: comparação entre as diferentes posiçöes. Rev Bras Med Esporte. 2002;8(2):32-6.

4. Nobrega MM, Tumiski JA, Jorge K, Worms RH, Rosa WM, Zanoni JHB, et al. A desidratação corporal de atletas amadores de futsal. Rev Bras Pres Fisiol Exercício. 2007:1 (5):24- 36.

5. Johann B, Deresz LF, Oliveira AM, Conde SR. Efeitos da suplementação de carboidratos sobre desempenho físico e metabólico em jogadores de futebol treinados e não treinados. Rev Bras Nutr Esp. 2015;9(54):544-52.

6. Silva MR, Carneiro CS, Crispim PAA, Melo NCS, Sales RR. Efeito do suplemento hidroeletrolítico na hidratação de jogadores juniores de futebol. Rev Bras Med Esporte. 2011;17(5):339-43.

7. Carvalho T, Mara TS. Hidratação e nutrição no esporte. Rev Bras Med Esporte. 2010;16(2):144-8

8. Casa DJ, Armstrong LE, Hillman SK, Mountain SJ, ReiffRV, Rich BSE, et al. NATA: National Athletics Trainer's Association Position Statement. J Athl Train. 2000;35(2):212-24.

9. Brito CJ. Hábitos de hidratação em judocas [dissertação]. Viçosa, MG: Universidade Federal de Viçosa, Departamento de Educação Física; 2002

10. Martins JCB, Ferreira FG. Nível de conhecimento dos atletas universitários da UFV sobre a hidratação. Fitness \& Performance Journal. 2005;4(3):175-85.

11. Rosa ASP. Comparação do perfil antropométrico: peso, altura e imc de atletas do santos futebol clube profissional e sub-20. Rev Bras Futsal Futebol. 2011;3(8):123-6.

12. Petreça DR, Ruske Neto HA. Perfil de composição corporal em atletas de base das modalidades de futsal e futebol de campo. Rev Saude Pesq. 2016;9(1):127-35.

13. Ersoy N, Ersoy G, Kutlu M. Assessment of hydration status of elite young male soccer players with different methods and new approach method of substitute urine strip. J Int Soc Sports Nutr. 2016;13(1):34

14. Motta MAS, Quintão DF. Nível de desidratação e estratégias nutricionais utilizadas antes edurante o treino de futebol de um grupo de adolescentes de Espera Feliz-Mg. Rev Bras Nutr Esp. 2016;10(59);518-23.

15. Mota GJ, Guerra I, Fernandes IW, Araújo PJ, Tavares CA. Nível de desidratação e temperatura corporal em jogadores de futebol de campo sub-18 durante um treino. Col Pesq Edu Fís. 2011;10(1):121-6.

16. Borusch E, Santos MCR, Guertzensten V, Zen VR, Silva SG. Desidratação em jogadores de futebol juniores. Rev Bras Nutr Esp. 2007;1(4):01-10.
17. Ravagnani FCP, Brandão CFC, PAz WB, Reis Filho AD, Fett CA, Ravagnani CFC. Perfil físico das diferentes posições de jogadores de futebol. Rev Bras Ci Mov.2013;21(2):11-8.

18. Costa IT, Silva JMG, Greco PJ, Mesquita I. Princípios táticos do jogo de futebol: conceitos e aplicação. Motriz. 2009;15(3):657-68.

19. Nery F, Guttierres PA, Dias CRM. Nível de desidratação após treinamento de ciclismo indoor. Rev Bras Med Esporte. 2014;20(4):320-5.

20. Pinto IFS, Berdacki SV, Biesek S.Avaliação da perda hídrica e do grau de conhecimento em hidratação de atletas de futebol americano. Rev Bras Nutr Esp. 2014;8(45):171-9.

21. Silverthorm DV. Fisiologia humana uma abordagem integrada. 5a ed. São Paulo: Artmed; 2010

22. Camargo MG, Furlan MMDP. Resposta fisiológica do corpo às temperaturas elevadas: exercício, extremos de temperatura e estresse térmico. Rev Saúd Pesq. 2011;4(2):278-88.

23. Drumond MG, Carvalho FR, Guimarães EMA. Hidratação em atletas adolescentes - hábitos e nível de conhecimento. Rev Bras Nutr Esp. 2007;1(2)76-93.

24. Silva RP, Mündel T, Natali AJ, Filho MGB, Lima JRP, Alfenas RCG, et al. Fluid balance of elite Brazilian youth soccer players during consecutive days of training. J Sport Sci. 2011;29(7):725-32.

25. Kurdak SS, Shirreffs SM, Maughan RJ, Ozgunen KT, Zeren C, Korkmaz S, et al. Hydration and sweating responses to hot-weather football competition. Scand J Med Sci Sports. 2010;20(Suppl 3):133-9.

26. Sociedade Brasileira de Medicina do Esporte (SBME). Modificações dietéticas, reposição hídrica, suplementos alimentares e drogas: comprovação de ação ergogênica e potenciais riscos para a saúde. Rev Bras Med Esporte. 2003;9(2):43-56.

27. Martins JCB, Ferreira FG. Nível de Conhecimento dos atletas universitários da UFV sobre hidratação. Fit Perf J. 2005;4(3):175-86.

28. Prado ES, Gonzaga WS, Dantas EHM. Conhecimento das práticas de hidratação dos atletas de vôlei de praia do estado de Sergipe. Rev Bras Ci Mov. 2010;18(3):29-34.

29. Ferreira FG, Altoé JL, Silva RP, Tsai LP, Fernandes A, Brito CJ, et al. Nível de conhecimento e práticas de hidratação em atletas de futebol de categoria de base. Rev Bras Cineantropom Desempenho Humano. 2009;11(2):202-9.

30. Hernandez AJ, Nahas RM. Modificações dietéticas, reposição hídrica suplementos alimentares e drogas: comprovação de ação ergogênica. Rev Bras Med Esporte. 2009;15(3):3-12. 